\title{
Manipulating the magnetization focal patterns using complex phase filters
}

\author{
M. UDHAYAKUMAR ${ }^{1}$, K. PRABAKARAN ${ }^{2}$, K.B. RAJESH ${ }^{1}$, \\ Z. JAROSZEWICZ ${ }^{3,4}$, D. VELAUTHAPILLAI ${ }^{5}$ \\ ${ }^{1}$ Department of Physics, Chikkanna Government Arts College, \\ Tiruppur, Tamilnadu, India \\ ${ }^{2}$ Department of Physics, Mahendra Arts and Science College (Autonomous), \\ Namakkal, Tamilnadu, India \\ ${ }^{3}$ Institute of Applied Optics, Department of Physical Optics, \\ Warsaw, Poland \\ ${ }^{4}$ National Institute of Telecommunications, Warsaw, Poland \\ ${ }^{5}$ Faculty of Engineering and Business Administration, \\ Western Norway University of Applied Sciences, Bergen, Norway \\ *Corresponding author: rajeskb@gmail.com
}

\begin{abstract}
Based on vector diffraction theory and inverse Faraday effect, the light induced magnetization distribution of a tightly focused azimuthally polarized Bessel-Gauss beam superimposed with a helical phase and modulated by an optimized multi belt complex phase filter (MBCPF) is analyzed numerically. It is noted that by adjusting the radii of different rings of the complex phase filter, one can achieve many novel magnetization focal distributions, such as sub-wavelength scale $(0.29 \lambda)$ and super-long $(71 \lambda)$ pure longitudinal magnetic probe and magnetization chain composed of nine, six and four magnetic spots of sub-wavelength scale. The authors expect that these results pave the path for fabricating magnetic lattices for spin wave operation, multiple atoms or magnetic particle trapping and transportation, confocal and magnetic resonance microscopy, as well as multilayer ultrahigh density magnetic storage.
\end{abstract}

Keywords: inverse Faraday effect, high NA lens, multi belt complex phase filter, azimuthally polarized Bessel-Gauss beam.

\section{Introduction}

In the past two decades, controlling the magnetic state of a medium with the help of femtosecond laser pulses is an emerging and rapidly developing research area in the field of modern magnetism. Recent research has shown that it is possible to realize deterministic and controllable switching of magnetic orders by ultrafast light pulses [ $\underline{1}-\underline{7}]$. Excitation of ultrafast magnetic oscillations through non-thermal optical processes has 
been demonstrated in many materials [ $\underline{8}-\underline{12}]$. The need to enhance the operation speed of modern electronic and magneto-optical (MO) devices, and to further develop the all-optical magnetic storage technology, has motivated many searches aimed at achieving a fundamental understanding of the mechanisms of magnetization dynamics and switching [1일 18 ]. Ultrafast optical laser pulses are currently used to manipulate the magnetization on the scale of a few hundred femtoseconds to picoseconds [19-23]. Unlike conventional magnetic storage devices, such an extremely fast and novel reversal mechanism does not require an external magnetic field, which provides us with an opportunity to write data with light. STANCIU et al. first demonstrated the all-optical magnetic recording (AOMR) by a single $40 \mathrm{fs}$ circularly polarized laser pulse by the inverse Faraday effect (IFE) [24]. To further facilitate those fascinating and practical applications, it is highly desirable to obtain a super-long and sub-wavelength longitudinal magnetization needle, as well as an extra-long and sub-wavelength longitudinal magnetization chain. Based on the vector diffraction theory and the inverse Faraday effect (IFE) in magneto-optic (MO) film, a sub-wavelength magnetic confinement under tight focusing of circularly polarized beam was demonstrated [25-29]. Recently, the light-induced magnetization produced by tight focusing of azimuthally polarized beams with helical phase has received considerable attention and a lot of research groups participated in studying the magnetization generated by the interaction between phase singularity and polarization singularity under the tight focusing condition [ $\underline{30}-\underline{32}]$. The pure longitudinal magnetization needle with a high aspect ratio above can be used to trap magnetic particles and realize high-density AOMR [ $\underline{33}, \underline{34}]$. However they might be less useful for a desired number of atoms trapping and transport, as well as multilayer magnetic-optical recoding and storage. Recently, NIE et al. proposed the possibility of obtaining a super-long $(12 \lambda)$ and sub-wavelength $(0.416 \lambda)$ longitudinal magnetization chain with single/dual channels in the focal region using 4Pi microscopy [하] ]. Later, using an azimuthally polarized vortex beam with proper amplitude modulation, Gong et al. found that a super-long (16 $\lambda$ ) magnetization chain, composed of 19 sub-wavelength $(0.44 \lambda)$ spherical spots of longitudinal magnetization field, can be achieved in the focal volume of the objective lenses for a $4 \pi$ tight focusing configuration [하]. However it is reported that the performance of $4 \mathrm{Pi}$ microscopy is significantly affected by aberration [37, 38]. Since the 4Pi microscopy can naively be considered as two separate microscopes configuration to operate in conjunction with each other, different combinations are associated with a phase shift of the interfering counter propagating waves and leads to significant different focal intensities. It is also noted that the parameters including polarization, chromatic aberrations, balance of intensities in the two arms and coherence of light have additional influence and need to be carefully considered in 4Pi configuration. Recently WEICHAO YAN et al. proposed that by selecting optimized parameters of a multi-Gaussian beam and topological charge of a spiral phase plate, not only a super-long and sub-wavelength longitudinal magnetization needle with single/dual channels for a single-lens high numerical aperture focusing system, but also an extra-long and three-dimensional super-resolution longitudinal magnetization chain with single/dual channels for a $4 \pi$ high numerical aperture focusing system can also 
be achieved in the focal region [39]. They also numerically investigated the light induced magnetization fields by azimuthally polarized vortex beam with a Gaussian annulus profile tightly focused with single and a pair of high NA lenses [40]. Recently, the same group reported that magnetization spot with FWHM of $0.37 \lambda$ and focal depth of $107 \lambda$ can be obtained using an azimuthally polarized vortex beam and a multi-zone phase filter. However, such a multi-zone plate phase filter is difficult to produce because of its complex phase transmittance from the point of view of the filter structure, and the performance of the complex phase filters are superior compared to other types of filter [41]. Recently we demonstrated the possibility of generating a sub-wavelength $(0.286 \lambda)$ super-long $(35 \lambda)$ magnetic needle and magnetic chain with multiple spherical longitudinal spots using an azimuthally polarized multi Gaussian vortex beam phase modulated by a specially designed complex phase filter and focused with high NA objective [42]. In this paper, we theoretically proposed a simple and novel method to generate a sub-wavelength super-long longitudinal magnetization needle and a spherical magnetization chain in the focal volume of the objective by focusing an azimuthally polarized Bessel-Gauss vortex (APBGV) beam phase modulated by a specially designed complex phase filter.

\section{Theory}

The schematic diagram is shown in Fig. 1a. An incident azimuthally polarized Bessel -Gauss beam (APBGB) travels through the vortex $0-2 \pi$ phase filter and becomes an azimuthally polarized Bessel-Gauss vortex beam (APBGVB). The APBGVB beam is then modulated with complex binary phase DOE with $N$ concentric belts. For a high NA lens, the electric field of the BG beam at the output pupil is defined as follows [43] :

$$
R(\theta)=J_{1}\left(2 \beta \frac{\sin \theta}{\sin \alpha}\right) \exp \left[-\left(\beta \frac{\sin \theta}{\sin \alpha}\right)^{2}\right]
$$

where $\beta$ is the parameter that denoted the ratio of pupil diameter to the beam diameter. Using the Debye approximation [4ㅗ $\underline{45}$ ] for tight focusing, we obtain the following ex-
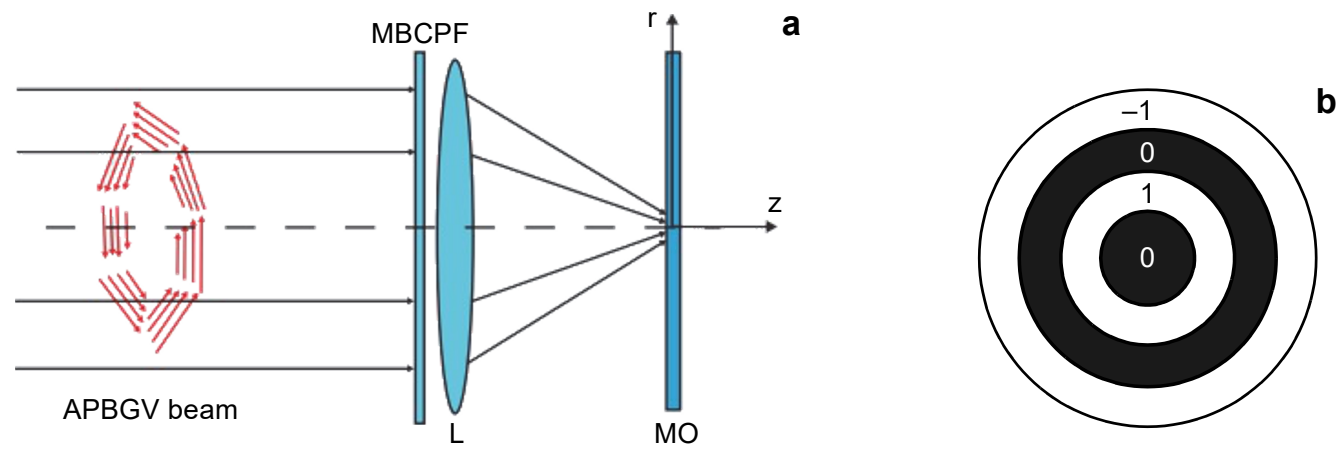

Fig. 1. The schematic diagram of a high NA focusing system is illustrated; a MO film is placed at the focal plane, which is illuminated by APBGV beam (a). Multi belt complex phase filter (MBCPF) (b). 
pression for the transverse components in the case of focusing an azimuthally polarized beam (as in this case, with no longitudinal component), having an $m$-th order vortex phase

$$
\begin{aligned}
& \mathbf{E}_{m, \perp}^{\mathrm{az}}(\rho, \varphi, z)=\left(\begin{array}{c}
E_{m, \rho}^{\mathrm{az}}(\rho, \varphi, z) \\
E_{m, \varphi}^{\mathrm{az}}(\rho, \varphi, z)
\end{array}\right) \\
& =\frac{1}{2} i^{m+1} k f \exp (i m \varphi) \\
& \quad \times \int_{0}^{\alpha} R(\theta) T(\theta)\left(\begin{array}{l}
J_{m+1}(k \rho \sin \theta)+J_{m-1}(k \rho \sin \theta) \\
-i\left[J_{m+1}(k \rho \sin \theta)-J_{m-1}(k \rho \sin \theta)\right]
\end{array}\right) \sin \theta \exp (i k z \cos \theta) \mathrm{d} \theta
\end{aligned}
$$

where $(\rho, \varphi, z)$ are the cylindrical coordinates in the focal region, $(\theta, \varphi)$ are the spherical angular coordinates of the focusing system's output pupil, $\alpha$ is the maximum value of the azimuthal angle related to the system's numerical aperture, $R(\theta)$ is the incident beam, $T(\theta)$ is the pupil's apodization function (equal to $(\cos \theta)^{1 / 2}$ for aplanatic systems), $k=2 \pi / \lambda$ is the wave number, $\lambda$ is the wavelength, and $f$ is the focal length. Equation (1) shows that the azimuthal polarization is retained only when focusing in the absence of a vortex phase $(m=0)$. When a vortex phase is present, part of the energy of the azimuthal component will be transferred to the orthogonal radial component. Based on the IFE, the magnetization field induced by tightly focusing azimuthally polarized beams with helical phase near the focal point is defined as [31]

$$
\mathbf{M}=i \gamma \mathbf{E} \times \mathbf{E}^{*}
$$

where $\mathbf{E}$ is the electric field, $\mathbf{E}^{*}$ is its conjugate, and $\gamma$ is a real constant proportional to the susceptibility of the material [느-4 $\underline{8}$ ]. By substituting Eqs. (2) into Eq. (3), the magnetization field can be given by

$$
\mathbf{M}=2 \gamma|A|^{2} \operatorname{Re}\left(E_{\rho} E_{\varphi}^{*}\right) \mathbf{e}_{z}
$$

Hence it is noted that the magnetization field induced by the tightly focusing azimuthally polarized vortex beam has only a longitudinal component.

\subsection{Focusing with multi belt complex phase filter (MBCPF)}

The combination of amplitude and phase only filters are usually called complex filters. Such filters are formed by cascading of phase filter and an amplitude filter. Recently, such filters are used to generate a 3D optical gauge and controllable 3D optical chain under the tight focusing condition [49]. The complex filter converge the optimized transverse super-resolution and also extends the depth of focus (DOF) and hence it in- 
tegrates the performance of two different filters onto a single filter and is expected to improve the performances of AOMR greatly. Recently, liquid crystal display spatial light modulators (LCD-SLMs) are used as programmable elements for complex modulation $[\underline{50}, \underline{51}]$. So, the goal of this paper is to give an investigation on the design and application of 4 belt complex phase filter (CPF) for AOMR system that extends the DOF and to realize transverse super-resolution of magnetization focal structure simultaneously, which will improve the performance of optical storage system greatly. Moreover we have optimized the CPF to generate a chain of spherical multiple magnetization spots for multiple trapping of magnetic particles. The four belt CPF is shown in Fig. 1b. The phase and amplitude function $A(\theta)$ of the multi belt complex phase filter $\operatorname{MBCPF}(\theta)$ is given by [드]

$$
\operatorname{MBCPF}(\theta)=\left\{\begin{aligned}
0 & \text { for } \quad 0<\theta<\theta_{1}, \quad \theta_{2}<\theta<\theta_{3} \\
1 & \text { for } \theta_{1}<\theta<\theta_{2} \\
-1 & \text { for } \theta_{3}<\theta<\theta_{\text {max }}
\end{aligned}\right.
$$

where $\theta_{1}$ and $\theta_{2}, \theta_{3}$ are radius of the first, second and the third zones, respectively. $\mathrm{A} \mathrm{MBCPF}$ is positioned at the pupil plane, where the transmissions from the inner to the outer zone belts are $0,1,0$, and -1 , respectively. The experimental realization of such a complex filter is much simpler than the filters proposed in the previous method [ $\underline{53}$ ]. Here we considered the 4-belt CPF and the set of four angles to obtain a particular focal patterns optimized by traditional global-search-optimization algorithm [ 54,55$]$. Based on this algorithm, we choose one structure with random values for $\theta_{1}$ to $\theta_{3}$ from all possibilities and simulate their focusing properties by vector diffraction theory and IFE. If the structure generates a sub-wavelength single focal spot and satisfies the limiting conditions that the FWHM of the generated magnetization segment is less than 0.5 , it is chosen as the initial structure during the optimization procedures. In the following steps, we continue to vary $\theta$ of one chosen zone to generate a uniform intensity on axial profile without affecting the limiting condition. We then repeat the procedure for $\theta$ of other zones. The same procedure is then adopted for creating multiple focal spots segments by considering the limiting conditions that the FWHM of each of the generated focal spot is less than $0.5 \lambda$ and there should be at least two, four or eight such focal spots in the focal segment

\section{Results and discussion}

Without loss of validity and generality, it was supposed that $\lambda=1$, and NA of the objective is 0.95 . For simplicity, here we assume that the refractive index $n=1, A=1$. The parameters used for the calculations are $m=1, \theta_{\max }=\arcsin (\mathrm{NA})$. For all calculations, the length unit is normalized to $\lambda$ and the energy density is normalized to unity. Figure 2 shows the magnetization distribution obtained for an azimuthally polarized APBGV beam with $\beta=2.5$ and 5.5. It is noted from Figs. $2 \mathbf{a}-2 \mathbf{c}$ when $\beta=2.5$ that the generated magnetization spot has FWHM of $0.74 \lambda$ and focal depth around $3.65 \lambda$. How- 

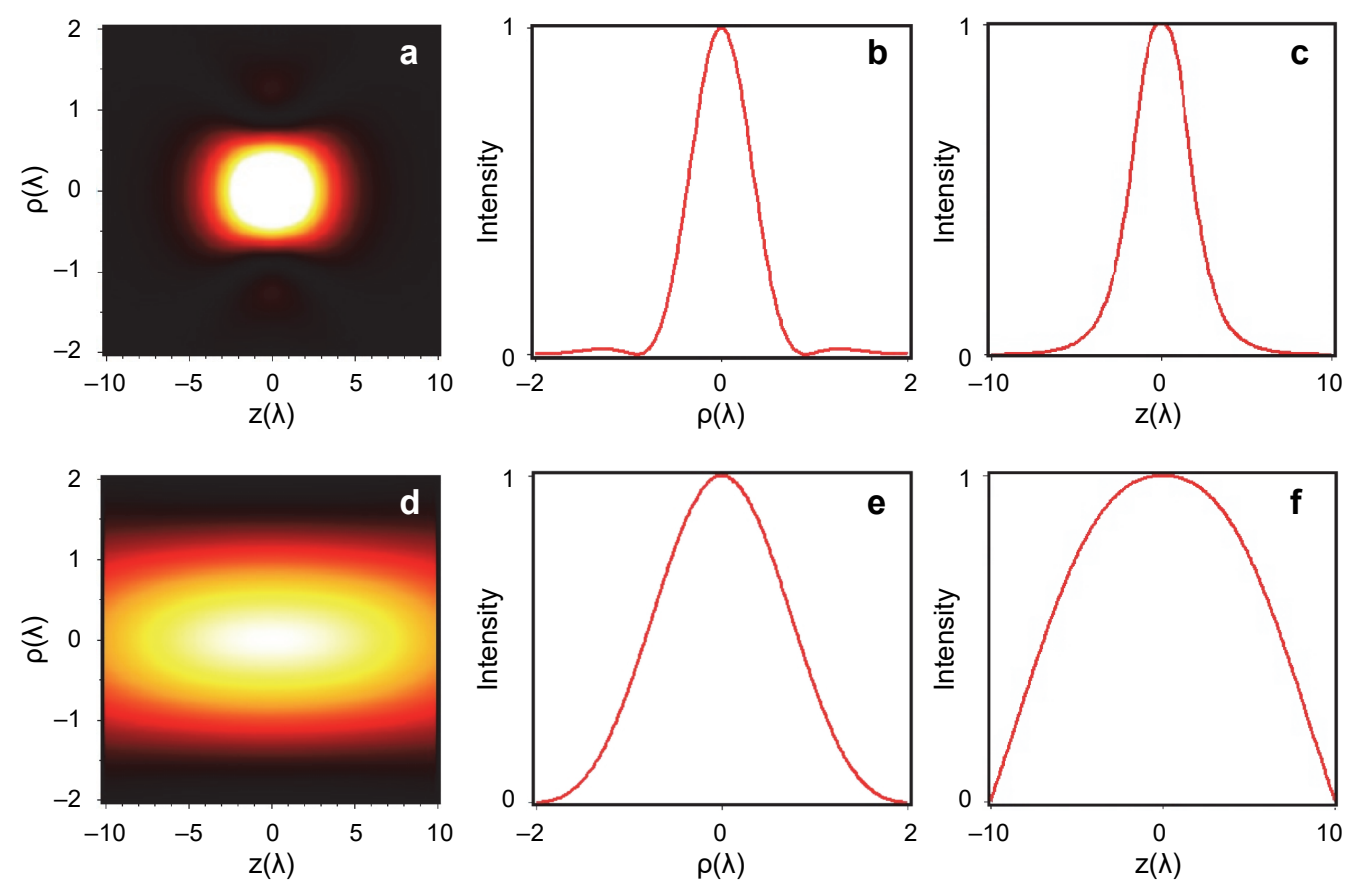

Fig. 2. Normalized magnetization distribution in $\rho$ - $z$ plane for APBGV beam (a,d), 2D normalized magnetization profiles along the radial direction at $z=0(\mathbf{b}, \mathbf{e})$, and magnetization profiles along the $z$-axis axial direction (c, f) for $\beta=2.5(\mathbf{a}, \mathbf{b}, \mathbf{c})$ and for $\beta=5.5(\mathbf{d}, \mathbf{e}, \mathbf{f})$.

ever we noted that increasing $\beta$ to 5.5 increased the focal depth as well as the spot size to $20 \lambda$ and $1.6 \lambda$, respectively, as shown in Figs. $2 \mathbf{d}-2 \mathbf{f}$. Thus to improve the focal depth without increasing the spot size, we proposed a MBCPF. The set of three angles of the 4 belt CPF optimized as an example for the above task using a traditional global search algorithm is $\theta_{1}=47.57^{\circ}, \theta_{2}=48.15^{\circ}, \theta_{3}=56.17^{\circ}, \alpha=71.84^{\circ}$. Figure 3 illustrated the focusing performance of a phase modulated azimuthally polarized Bessel-Gauss beam with $\beta=2.5$ and 5.5 .

Figures 3a-3c shows that the above optimizing MBCPF generates an axial chain of sub-wavelength scale nine magnetization spots, each having FWHM of $0.326 \lambda$, DOF of $1.3 \lambda$ and are separated by axial distance $1.3 \lambda$ when $\beta=2.5$. Such a pure magnetization chain found potential significance in multilayer magneto-optical data, ultra-compact MO devices, magnetic particle trapping and transportation, fabricating magnetic lattices for spin wave operation, as well as confocal and magnetic resonance [ $\underline{56}-\underline{59}]$. It is also noted from Figs. $3 \mathbf{d}-3 \mathbf{f}$ that the above optimized MBCPF also generates a magnetization segment confined with FWHM $0.29 \lambda$ and extends axially without diffraction up to $71 \lambda$ when $\beta=5.5$. Such a pure magnetization probe with extended focal depth may find applications in AOMR and in particle trapping. Moreover, the DOF achieved is much larger than the previously proposed methods and is compared in the Table. 

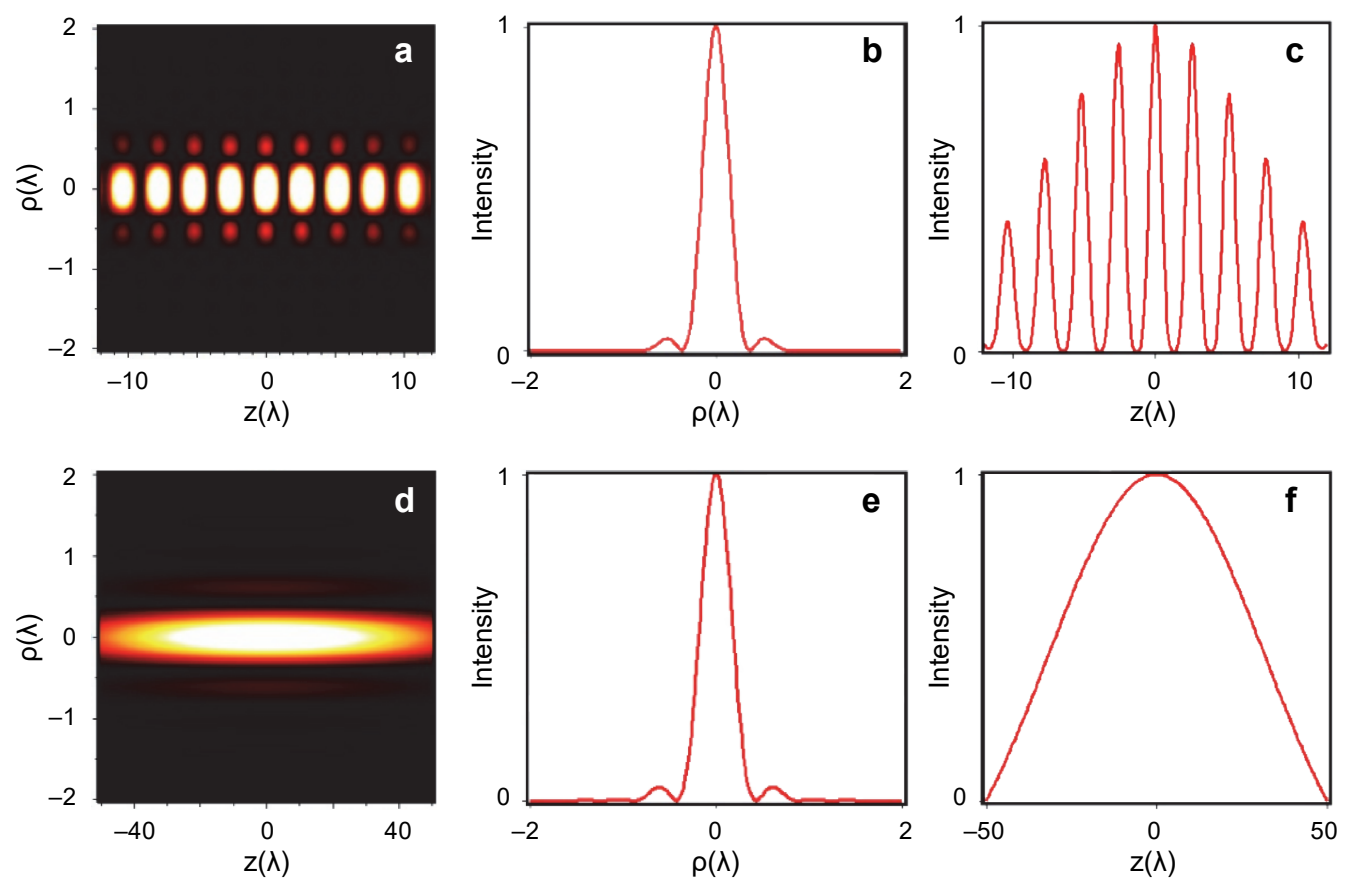

Fig. 3. Normalized magnetization distribution in $\rho-z$ plane for phase modulated APBGV beam (a, d), 2D normalized magnetization profiles along the radial direction $(\mathbf{b}, \mathbf{e})$, and magnetization profiles along the $z$-axis $(\mathbf{c}, \mathbf{f})$ for $\beta=2.5(\mathbf{a}, \mathbf{b}, \mathbf{c})$ and for $\beta=5.5(\mathbf{d}, \mathbf{e}, \mathbf{f})$.

$\mathrm{T}$ a b 1 e. Comparison of performance of the proposed configuration with similar configurations.

\begin{tabular}{|c|c|c|}
\hline Methods & FWHM & DOF \\
\hline Binary phase filter [31] & $0.38 \lambda$ & $7.48 \lambda$ \\
\hline Vortex binary filters $[\underline{35}]$ & $0.416 \lambda$ & $12 \lambda$ \\
\hline 4pi method $[\underline{36}]$ & $0.44 \lambda$ & $16 \lambda$ \\
\hline Azimuthally polarized annular multi Gaussian [42] & $0.286 \lambda$ & $35 \lambda$ \\
\hline Azimuthally polarized DG beam [ $\underline{59}]$ & $0.29 \lambda$ & $52.2 \lambda$ \\
\hline Proposed method (APBGVB) & $0.29 \lambda$ & $71 \lambda$ \\
\hline
\end{tabular}

Figure 4 shows that MBCPF optimized with angles $\theta_{1}=43.5^{\circ}, \theta_{2}=48.15^{\circ}, \theta_{3}=$ $56.17^{\circ}, \alpha=71.84^{\circ}$ can generate a chain of six magnetization spots axially separated by a distance of $1.6 \lambda$ and each having FWHM of $0.30 \lambda$ with DOF as $1.8 \lambda$ when $\beta=2.5$. We further noted that further increasing $\beta$ as 5.5 can generate a magnetization spot having FWHM of $0.32 \lambda$ and focal depth $27 \lambda$. Figures $5 \mathbf{a}-5 \mathbf{c}$ shows that the MBCPF generates an axial chain of sub-wavelength four magnetization spots each having FWHM of $0.31 \lambda$, DOF of $1.8 \lambda$ and separated by axial distance $1.6 \lambda$, when $\beta=2.5$. We further noted that further increasing to $\beta=5.5$ can generate a magnetization spot having FWHM of 0.34 and focal depth $12.4 \lambda$. The set of angles of a complex phase filter (CPF) 

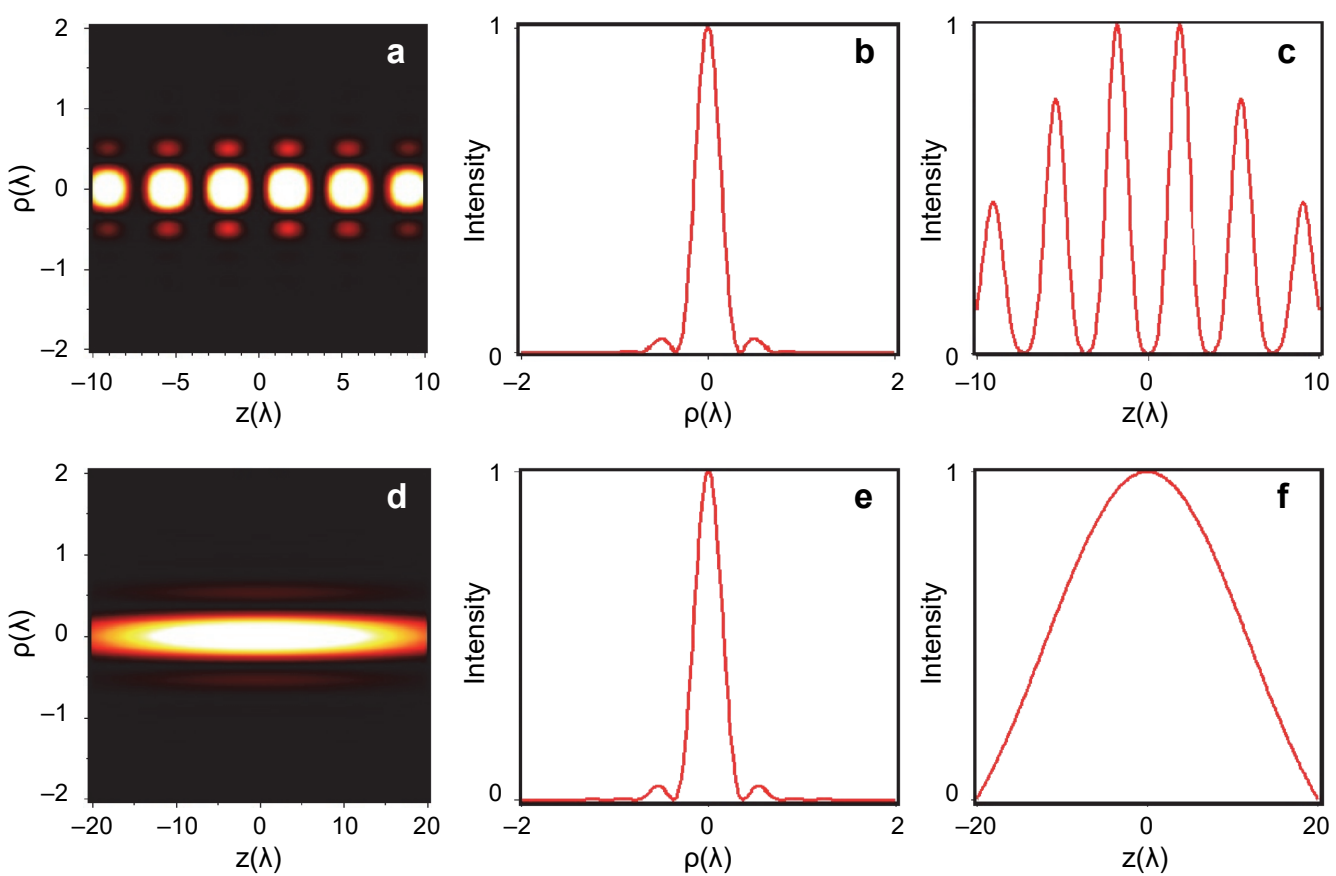

Fig. 4. The same as Fig.3 but for MBCPF optimized with different set of angles.
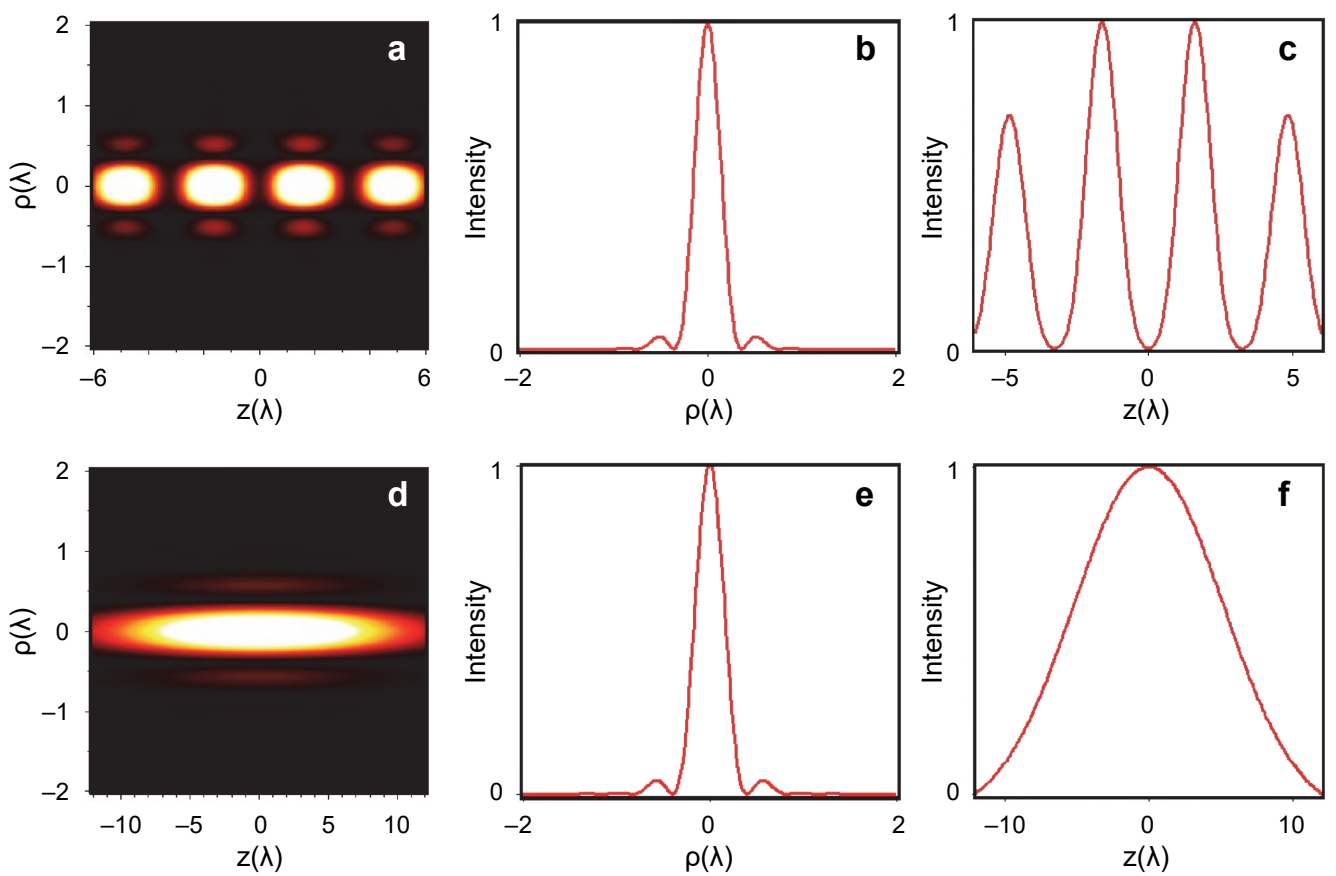

Fig. 5. The same as Fig. 3 but for MBCPF optimized with different set of angles. 

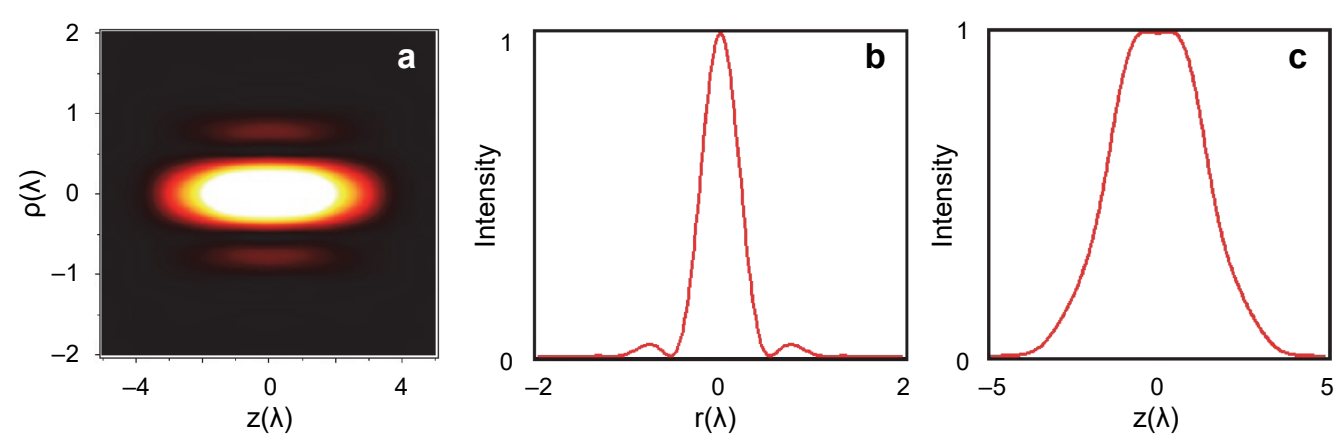

Fig. 6. Normalized magnetization distribution in $\rho$-z plane for APBGV beam (a), 2D normalized magnetization profiles along the radial direction at $z=0(\mathbf{b})$, and magnetization profiles along the $z$-axis axial direction (c) for $\beta=1.6$.

optimized for the above mentioned focal segments is $\theta_{1}=43.5^{\circ}, \theta_{2}=47^{\circ}, \theta_{3}=56.17^{\circ}$, $\alpha=71.84^{\circ}$. Thus from the above example we showed that with a perfectly optimized MBCPF one can tune the magnetization focal structure from a segment of multiple spherical magnetization spots of specific numbers to a magnetization probe of fixed focal depth by properly tuning the pupil to beam ratio $\beta$ of incident APBGV beam from 2.5 to 5.5. Apart from this, Fig. 6a shows the possibility of generating a sub-wavelength scale magnetization probe with fine axial homogeneity generated by MBCPF optimized with angles $\theta_{1}=24.07^{\circ}, \theta_{2}=42.42^{\circ}, \theta_{3}=56.17^{\circ}, \alpha=71.84^{\circ}$ for $\beta=1.6$. Figure $6 \mathbf{b}$ shows that the FWHM of the generated magnetic probe is $0.47 \lambda$. The axial intensity distribution shows in Fig. $6 \mathbf{c}$ that the generated magnetization focal segment is axially homogenous and its FWHM the DOF is around 3.36 $\lambda$. Thus, by using a single focusing unit and by properly modulating the phase of incident APBGV beam using optimizing MBCPF, one can generate a super-resolution magnetic probe of extended focal depth suitable AOMR and a chain of multiple magnetization spots for multiple trapping of magnetic particles.

\section{Conclusion}

In conclusion, the proposed multi belt complex phase modulation scheme with high NA lens for tightly focused azimuthally polarized Bessel-Gauss beam superimposed with a helical phase and modulated by an optimized multi belt complex phase filter (MBCPF) is analyzed numerically. It is noted that by adjusting the radii of different rings of the complex phase filter, one can achieve many novel magnetization focal distributions, such as a sub-wavelength scale $(0.29 \lambda)$ and super-long $(71 \lambda)$ longitudinal magnetic probe and multiple magnetization spots suitable for all-optical magnetic recording, and magnetic lattices for spin wave operation and atomic trapping are generated.

Acknowledgments - This work is supported by the Department of Science and Technology (DST), India, under project SERB-YSS (F.No. 2015/001852). 


\section{References}

[1] Reber A., Hohlfeld J., The magneto-optical Barnett effect: circularly polarized light induced femtosecond magnetization reversal, Physics Letters A 372(11), 2008, pp. 1915-1918, DOI: 10.1016/j.phys leta.2007.10.048.

[2] Stupakiewicz A., Szerenos K., Afanasiev D., Kirilyuk A., Kimel A.V., Ultrafast nonthermal photo-magnetic recording in a transparent medium, Nature 542, 2017, pp. 71-74, DOI: $10.1038 /$ nature20807.

[3] Zhang L., Takahashi Y.K., Hono K., Stipe B.C., Juang J.-Y., Grobis M., L1 $1_{0}$-ordered FePtAg-C granular thin film for thermally assisted magnetic recording media (invited), Journal of Applied Physics 109(7), 2011, article ID 07B703, DOI: 10.1063/1.3536794.

[4] Savoini M., Medapalli R., Koene B., Khorsand A.R., Le Guyader L., Duò L., Finazzi M., Tsukamoto A., Itoh A., Nolting F., Kirilyuk A., Kimel A.V., Rasing Th., Highly efficient all-optical switching of magnetization in GdFeCo microstructures by interference-enhanced absorption of light, Physical Review B 86(14), 2012, article ID 140404(R), DOI: 10.1103/PhysRevB.86.140404.

[5] Varaprasad B.S.D.Ch.S., Chen M., Takahashi Y.K., Hono K., L1 $1_{0}$-ordered FePt-based perpendicular magnetic recording media for heat-assisted magnetic recording, IEEE Transactions on Magnetics 49(2), 2013, pp. 718-722, DOI: 10.1109/TMAG.2012.2218227.

[6] Hassdenteufel A., Hebler B., Schubert C., Liebig A., Teich M., Helm M., Aeschlimann M.A.M., BRATSCHITSCH R., Thermally assisted all-optical helicity dependent magnetic switching in amorphous $\mathrm{Fe}_{100-x} \mathrm{~Tb}_{x}$ alloy films, Advanced Materials 25(22), 2013, pp. 3122-3128, DOI: 10.1002/adma. 201300176.

[7] Li Zhang, Takahashi Y.K, Perumal A., Hono K., L1 $1_{0}$-ordered high coercivity (FePt)Ag-C granular thin films for perpendicular recording, Journal of Magnetism and Magnetic Materials 322(18), 2010, pp. 2658-2664, DOI: 10.1016/j.jmmm.2010.04.003.

[8] Kimel A.V., Kirilyuk A., Tsvetkov A., Pisarev R.V., Rasing Th., Laser induced ultrafast spin reorientation in the antiferromagnetic $\mathrm{TmFeO}_{3}$, Nature 429, 2004, pp. 850-853, DOI: 10.1038/nature02659.

[9] Stanciu C.D., Kimel A.V., Hansteen F., Tsukamoto A., Itoh A., Kirilyuk A., Rasing Th., Ultrafast spin dynamics across compensation points in ferrimagnetic $G d F e C o$ : the role of angular momentum compensation, Physical Review B 73(22), 2006, article ID 220402(R), DOI: 10.1103/PhysRevB.73. 220402.

[10] Tian-Min Liu, Tianhan Wang, Reid A.H., Savoini M., Xiaofei Wu, Koene B., Granitzka P., Graves C.E., Higley D.J., Zhao Chen, Razinskas G., Hantschmann M., Scherz A., Stöhr J., Tsukamoto A., Hecht B., Kimel A.V., Kirilyuk A., Rasing T., Dürr H.A., Nanoscale confinement of all-optical magnetic switching in TbFeCo-competition with nanoscale heterogeneity, Nano Letters 15(10), 2015, pp. 6862-6868, DOI: 10.1021/acs.nanolett.5b02743.

[11] Ellis M.O.A., Fullerton E.E., Chantrell R.W., All-optical switching in granular Ferro magnets caused by magnetic circular dichroism, Scientific Reports 6, 2016, p. 30522, DOI: 10.1038/srep30522.

[12] Tao Huang, Haiwei Wang, Weiming Cheng, Yuhao Zou, Changsheng Xie, Critical switching fluence of $L 1_{0}-\mathrm{FePt}$ nanoparticles with practical size to ultrafast all-optical polarization switching, EPL 118(6), 2017, article ID 67006, DOI: 10.1209/0295-5075/118/67006.

[13] Hohlfeld J., Matthias E., Knorren R., Bennemann K.H., Nonequilibrium magnetization dynamics of nickel, Physical Review Letters 78(25), 1997, pp. 4861-4864, DOI: 10.1103/PhysRevLett.78.4861.

[14] Malinowski G., Dalla Longa F., Rietjens J.H., Paluskar P.V., Huijink R., Swagten H.J.M., Koopmans B., Control of speed and efficiency of ultrafast demagnetization by direct transfer of spin angular momentum, Nature Physics 4(11), 2008, pp. 855-858, DOI: 10.1038/nphys1092.

[15] Battiato M., Carva K., Oppeneer P.M., Superdiffusive spin transport as a mechanism of ultrafast demagnetization, Physical Review Letters 105(2), 2010, article ID 027203, DOI: 10.1103/PhysRev Lett.105.027203. 
[16] Tianqi Li, Patz A., Mouchliadis L., Jiagiang Yan, Lograsso T.A., Perakis I.E., Jigang Wang, Femtosecond switching of magnetism via strongly correlated spin-charge quantum excitations, Nature 496, 2013, pp. 69-73, DOI: 10.1038/nature11934.

[17] Kirilyuk A., Kimel A.V., Rasing Th., Laser-induced magnetization dynamics and reversal in ferromagnetic alloys, Reports on Progress in Physics 76(2), 2013, article ID 026501, DOI: $10.1088 /$ 0034-4885/76/2/026501.

[18] El Hadri M.S., Pirro P., Lambert C.-H., Petit-Watelot S., Quessab Y., Hehn M., Montaigne F., Malinowski G., Mangin S., Two types of all-optical magnetization switching mechanisms using femtosecond laser pulses, Physical Review B 94(6), 2016, article ID 064412, DOI: 10.1103/PhysRevB. 94.064412 .

[19] Gerrits Th., van den Berg H.A.M., Hohlfeld J., Bär L., Rasing Th., Ultrafast precessional magnetization reversal by picosecond magnetic field pulse shaping, Nature 418, 2002, pp. 509-512, DOI: $10.1038 /$ nature00905.

[20] Thiele J.-U., Buess M., BACK C.H., Spin dynamics of the antiferromagnetic-to-ferromagnetic phase transition in FeRh on a sub-picosecond time scale, Applied Physics Letters 85(14), 2004, pp. 2857 -2959, DOI: 10.1063/1.1799244.

[21] Kimel A.V., Kirilyuk A., Rasing T., Femtosecond opto-magnetism: ultrafast laser manipulation of magnetic materials, Laser and Photonics Reviews 1(3), 2007, pp. 275-287, DOI: 10.1002/lpor. 200710022.

[22] Zhang G.P., HüBner W., Lefkidis G., Yihua Bai, George T.F., Paradigm of the time-resolved magneto-optical Kerr effect for femtosecond magnetism, Nature Physics 5, 2009, pp. 499-502, DOI: $10.1038 /$ nphys 1315.

[23] Le Guyader L., Savoini M., El Moussaoui S., Buzzi M., Tsukamoto A., Itoh A., Kirilyuk A., Rasing T., Kimel A.V., Nolting F., Nanoscale sub-100 picosecond all-optical magnetization switching in GdFeCo microstructures, Nature Communications 6, 2015, article ID 5839, DOI: $10.1038 /$ ncomms6839.

[24] Stanciu C.D., Hansteen F., Kimel A.V., Kirilyuk A., Tsukamoto A., Itoh A., Rasing Th., All-optical magnetic recording with circularly polarized light, Physical Review Letters 99(4), 2007, article ID 047601, DOI: 10.1103/PhysRevLett.99.047601.

[25] YaOJu Zhang, JiAnPING BAI, High-density all-optical magnetic recording using a high-NA lens illuminated by circularly polarized pulse lights, Physics Letters A 372(41), 2008, pp. 6294-6297, DOI: 10.1016/j.physleta.2008.08.048.

[26] ZhUANG YOU-YI, ZHANG YAO-JU, Improving recording density of all-optical magnetic storage by using high-pass angular spectrum filters, Chinese Physics Letters 26(10), 2009, article ID 108501.

[27] Yaoju Zhang, Yoichi Okuno, Xun Xu, All-optical magnetic superresolution with binary pupil filters, Journal of the Optical Society of America B 26(7), 2009, pp. 1379-1383, DOI: 10.1364/ JOSAB.26.001379.

[28] Yaoju Zhang, JiAnPING BaI, Theoretical study on all-optical magnetic recording using a solid immersion lens, Journal of the Optical Society of America B 26(1), 2009, pp. 176-182, DOI: 10.1364/ JOSAB.26.000176.

[29] Ravi V., Suresh P., Rajesh K.B., Jaroszewicz Z., Anbarasan P.M., Pillai T.V.S., Generation of sub-wavelength longitudinal magnetic probe using high numerical aperture lens axicon and binary phase plate, Journal of Optics 14(5), 2012, article ID 055704, DOI: 10.1088/2040-8978/14/5/055704.

[30] Yunshan Jiang, XiangPing Li, Min Gu, Generation of sub-diffraction-limited pure longitudinal magnetization by the inverse Faraday effect by tightly focusing an azimuthally polarized vortex beam, Optics Letters 38(16), 2013, pp. 2957-2960, DOI: 10.1364/OL.38.002957.

[31] Sicong Wang, Xiangping Li, Jianying Zhou, Min Gu, Ultralong pure longitudinal magnetization needle induced by annular vortex binary optics, Optics Letters 39(17), 2014, pp. 5022-5025, DOI: 10.1364/OL.39.005022. 
[32] Sicong Wang, Xiangping Li, Jianying Zhou, Min Gu, All-optically configuring the inverse Faraday effect for nanoscale perpendicular magnetic recording, Optics Express 23(10), 2015, pp. 13530-13536, DOI: 10.1364/OE.23.013530.

[33] Wangzi Ma, Dawei Zhang, Linwei Zhu, Jiannong Chen, Super-long longitudinal magnetization needle generated by focusing an azimuthally polarized and phase-modulated beam, Chinese Optics Letters 13(5), 2015, article ID 052101.

[34] Atutov S.N., Calabrese R., Guidi V., Mai B., Rudavets A.G., Scansani E., Tomassetti L., Biancalana V., Burchianti A., Marinelli C., Mariotti E., Moi L., Veronesi S., Fast and efficient loading of a Rb magneto-optical trap using light-induced atomic desorption, Physical Review A 67(5), 2003, article ID 053401, DOI: 10.1103/PhysRevA.67.053401.

[35] Zhongquan Nie, Weigiang Ding, Dongyu Li, Xueru Zhang, Yuxiao Wang, Yinglin Song, Spher$i c a l$ and sub-wavelength longitudinal magnetization generated by $4 \pi$ tightly focusing radially polarized vortex beams, Optics Express 23(2), 2015, pp. 690-701, DOI: 10.1364/OE.23.000690.

[36] Liping Gong, Lu Wang, Zhuqing Zhu, Xiaolei Wang, Hua Zhao, Bing Gu, Generation and manipulation of super-resolution spherical magnetization chains, Applied Optics 55(21), 2016, pp. 5783-5789, DOI: 10.1364/AO.55.005783.

[37] Gould T.J., Burke D., Bewersdorf J., Booth M.J., Adaptive optics enables 3D STED microscopy in aberrating specimens, Optics Express 20(19), 2012, pp. 20998-21009, DOI: 10.1364/OE.20.020998.

[38] Xiang Hao, Antonello J., Allgeyer E.S., Bewersdorf J., Booth M.J., Aberrations in 4Pi microscopy, Optics Express 25(13), 2017, pp. 14049-14058, DOI: 10.1364/OE.25.014049.

[39] Weichao Yan, Zhongquan Nie, Xueru Zhang, Yuxiao Wang, Yinglin Song, Magnetization shaping generated by tight focusing of azimuthally polarized vortex multi-Gaussian beam, Applied Optics 56(7), 2017, pp. 1940-1946, DOI: 10.1364/AO.56.001940.

[40] Weichao Yan, Zhongquan Nie, Xueru Zhang, Yuxiao Wang, Yinglin Song, Theoretical guideline for generation of an ultra-long magnetization needle and a super-long conveyed spherical magnetization chain, Optics Express 25(19), 2017, pp. 22268-22279, DOI: 10.1364/OE.25.022268.

[41] Weichao Yan, Zhongquan Nie, Xueru Zhang, Yuxiao Wang, Yinglin Song, Generation of an ultralong pure longitudinal magnetization needle with high axial homogeneity using an azimuthally polarized beam modulated by pure multi-zone plate phase filter, Journal of Optics 19(8), 2017, article ID 085401, DOI: 10.1088/2040-8986/aa73ce.

[42] Udhayakumar M., Prabakaran K., Rajesh K.B., Jaroszewicz Z., Belafhal A., Generating sub wavelength pure longitudinal magnetization probe and chain using complex phase plate, Optics Communications 407, 2018, pp. 275-279, DOI: 10.1016/j.optcom.2017.09.007.

[43] Yew E.Y.S., ShepPard C.J.R., Tight focusing of radially polarized Gaussian and Bessel-Gauss beams, Optics Letters 32(23), 2007, pp. 3417-3419, DOI: 10.1364/OL.32.003417.

[44] Richards B., Wolf E., Electromagnetic diffraction in optical systems. II. Structure of the image field in an aplanatic system, Proceedings of the Royal Society of London. Series A. Mathematical and Physical Sciences 253(1274), 1959, pp. 358-379, DOI: 10.1098/rspa.1959.0200.

[45] Khonina S.N., Kazanskiy N.L., Volotovsky S.G., Vortex phase transmission function as a factor to reduce the focal spot of high-aperture focusing system, Journal of Modern Optics 58(9), 2011, pp. 748-760, DOI: 10.1080/09500340.2011.568710.

[46] Pershan P.S., Nonlinear optical properties of solids: energy considerations, Physical Review 130(3), 1963, p. 919, DOI: 10.1103/PhysRev.130.919.

[47] Van der Ziel J.P., Pershan P.S., Malmstrom L.D., Optically-induced magnetization resulting from the inverse Faraday effect, Physical Review Letters 15(5), 1965, p. 190, DOI: 10.1103/PhysRevLett. 15.190.

[48] Pershan P.S., van der Ziel J.P., Malmstrom L.D., Theoretical discussion of the inverse Faraday effect, Raman scattering, and related phenomena, Physical Review 143(2), 1966, p. 574, DOI: $10.1103 /$ PhysRev.143.574. 
[49] Hanming Guo, Xiaoyu Weng, Xiangmei Dong, Guorong Sui, Xiumin Gao, Songlin Zhuang, Three dimensional optical cage formed by TEM $M_{01}$ mode radially polarized Laguerre-Gaussian beam, Journal of Optics 40(4), 2011, pp. 206-212, DOI: 10.1007/s12596-011-0055-8.

[50] Jianwei Cao, Qingkui Chen, Hanming Guo, Creation of a controllable three dimensional optical chain by $T E M_{01}$ mode radially polarized Laguerre-Gaussian beam, Optik 124(15), 2013, pp. 2033-2036, DOI: $10.1016 /$ j.ijleo.2012.06.057.

[51] Gundu P.N., Hack E., Rastogi P., Superspeckles: a new application of optical superresolution, Optics Express 13(17), 2005, pp. 6468-6475, DOI: 10.1364/OPEX.13.006468.

[52] Prabakaran K., Rajesh K.B., Pillai T.V.S., Generation of multiple sub wavelength focal spot segments using radially polarized Bessel Gaussian beam with complex phase filter, Optik 125(13), 2014, pp. 3159-3161, DOI: 10.1016/j.ijleo.2013.12.009.

[53] Gundu P.N., Hack E., Rastogi P., High efficient superresolution combination filter with twin LCD spatial light modulators, Optics Express 13(8), 2005, pp. 2835-2842, DOI: 10.1364/OPEX.13.002835.

[54] Li Liu, GuiYIng WANG, Designing superresolution optical pupil filter with constrained global optimization algorithm, Optik 119(10), 2008, pp. 481-484, DOI: 10.1016/j.ijleo.2007.03.001.

[55] Haifeng Wang, Luping Shi, Lukyanchuk B., Sheppard C., Chong Tow Chong, Creation of a needle of longitudinally polarized light in vacuum using binary optics, Nature Photonics 2, 2008, pp. 501 -505, DOI: 10.1038/nphoton.2008.127.

[56] Khorsand A.R., Savoini M., Kirilyuk A., Kimel A.V., Tsukamoto A., Itoh A., Rasing Th., Role of magnetic circular dichroism in all-optical magnetic recording, Physical Review Letters 108(12), 2012, article ID 127205, DOI: 10.1103/PhysRevLett.108.127205.

[57] Majors P.D., Minard K.R.E.J., Ackerman G.R., Holtom D.F., Hopkins C.I., Parkinson Weber T.J., WIND R.A., A combined confocal and magnetic resonance microscope for biological studies, Review of Scientific Instruments 73(12), 2002, pp. 4329-4338, DOI: 10.1063/1.1517146.

[58] Grinolds M.S., Warner M., De Greve K., Dovzhenko Y., Thiel L., Walsworth R.L., Hong S., Maletinsky P., Yacoby A., Subnanometre resolution in three-dimensional magnetic resonance imaging of individual dark spins, Nature Nanotechnology 9(4), 2014, pp. 279-284, DOI: $\underline{10.1038 / \text { nnano.2 }}$ $\underline{014.30 .}$

[59] Udhayakumar M., Prabakaran K., Rajesh K.B., Jaroszewicz Z., A. Belafhal, D. Velauthapillai, Generation of ultra-long pure magnetization needle and multiple spots by phase modulated doughnut Gaussian beam, Optics and Laser Technology 102, 2018, pp. 40-46, DOI: 10.1016/j.optlastec. 2017.12.008.

Received July 3, 2018

in revised form October 3, 2018 